\title{
Testing a new method for scattering in finite volume in the $\phi^{4}$ theory
}

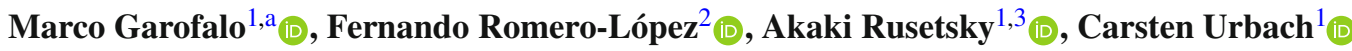 \\ ${ }^{1}$ HISKP (Theory), Rheinische Friedrich-Wilhelms-Universität Bonn, Nussallee 14-16, 53115 Bonn, Germany \\ ${ }^{2}$ IFIC, CSIC-Universitat de València, 46980 Paterna, Spain \\ 3 Tbilisi State University, 0186 Tbilisi, Georgia
}

Received: 30 July 2021 / Accepted: 15 November 2021 / Published online: 26 November 2021

(C) The Author(s) 2021

\begin{abstract}
We test an alternative proposal by Bruno and Hansen (J High Energy Phys 2021(6), https://doi.org/10. 1007/JHEP06(2021)043, 2021) to extract the scattering length from lattice simulations in a finite volume. For this, we use a scalar $\phi^{4}$ theory with two mass nondegenerate particles and explore various strategies to implement this new method. We find that the results are comparable to those obtained from the Lüscher method, with somewhat smaller statistical uncertainties at larger volumes.
\end{abstract}

\section{Introduction}

Lattice QCD has been shown to be a powerful tool to determine scattering quantities from first principles. The standard approach is the Lüscher method [2], which relates the finitevolume spectrum obtained from the lattice to the infinitevolume scattering amplitude. It has been applied to many physical systems, including results at the physical point-see Ref. [3] for a review. The formalism has also been recently extended to three particles with three different but conceptually equivalent formulations available in the literature at present [4-8], see Refs. [9,10] for recent reviews.

In Ref. [1], the authors propose a new strategy to extract scattering quantities. Henceforth, this will be referred to as the BH method. This approach is based on the usage of fourpoint functions rather than energy levels. The hope is that this approach can be generalised more easily to multi-hadron processes.
As pointed out by the authors, the case of threshold kinematics is particularly favourable as it allows for a direct extraction of the scattering length, with the $\pi N$ channel being one concrete example.

In this letter we test this novel approach in a scalar $\phi^{4}$ theory. Using this theory has proven to be an excellent test bed for novel scattering studies, as shown in Refs. [11-13]. In order to mimic the $\pi N$ case, we consider two mass nondegenerate real scalar particles. We explore the necessary techniques, and the optimal approach to use the $\mathrm{BH}$ method at threshold. Moreover, we compare to the standard Lüscher approach and find good agreement.

\section{Description of the Model}

The Euclidean model used here is composed by two real scalar fields $\phi_{i}, i=0,1$ with the Lagrangian

$$
\mathcal{L}=\sum_{i=0,1}\left(\frac{1}{2} \partial_{\mu} \phi_{i} \partial_{\mu} \phi_{i}+\frac{1}{2} m_{i} \phi_{i}^{2}+\lambda_{i} \phi_{i}^{4}\right)+\mu \phi_{0}^{2} \phi_{1}^{2}
$$

with nondegenerate (bare) masses $m_{0}<m_{1}$. The Lagrangian has a $Z_{2} \otimes Z_{2}$ symmetry $\phi_{0} \rightarrow-\phi_{0} \otimes \phi_{1} \rightarrow-\phi_{1}$, which prevents sectors with even and odd number of particles to mix.

To study the problem numerically, we define the theory on a finite hypercubic lattice with lattice spacing $a$ and a volume $T \cdot L^{3}$, where $T$ denotes the Euclidean time length and $L$ the spatial length. We define the derivatives of the Lagrangian (Eq. 1) on a finite lattice as the finite differences $\partial_{\mu} \phi(x)=\frac{1}{a}(\phi(x+a \mu)-\phi(x))$. In addition, periodic boundary conditions are assumed in all directions. The discrete action is given in Ref. [12] for the complex scalar theory, but it is trivial to adapt to this case. We set $a=1$ in the following for convenience.

\footnotetext{
a e-mail: garofalo@ hiskp.uni-bonn.de (corresponding author)
} 


\section{Observables}

In Ref. [1], Bruno and Hansen derived a relation between the scattering length $a_{0}$ and the following combination of Euclidean four-point and two-point correlation functions at the two-particle threshold:

$C_{4}^{\mathrm{BH}}\left(t_{f}, t, t_{i}\right) \equiv \frac{\left\langle\tilde{\phi}_{0}\left(t_{f}\right) \tilde{\phi}_{1}(t) \tilde{\phi}_{1}\left(t_{i}\right) \tilde{\phi}_{0}(0)\right\rangle}{\left\langle\tilde{\phi}_{0}\left(t_{f}\right) \tilde{\phi}_{0}(0)\right\rangle\left\langle\tilde{\phi}_{1}(t) \tilde{\phi}_{1}\left(t_{i}\right)\right\rangle}-1$,

with the time ordering $t_{f}>t>t_{i}>0$, and $\tilde{\phi}_{i}(t)=$ $\sum_{\mathbf{x}} \phi_{i}(t, \mathbf{x})$ being field projected to zero spatial momentum. The relation of $C_{4}^{\mathrm{BH}}$ to the scattering length reads

$$
\begin{aligned}
& C_{4}^{\mathrm{BH}}\left(t_{f}, t, t_{i}\right) \stackrel{T \gg t_{f} \gg t}{\longrightarrow} \frac{2}{L^{3}}\left[\pi \frac{a_{0}}{\mu_{01}}\left(t-t_{i}\right)\right. \\
&\left.-2 a_{0}^{2} \sqrt{\frac{2\left(t-t_{i}\right)}{\mu_{01}}}+O\left(\left(t-t_{i}\right)^{0}\right)\right],
\end{aligned}
$$

where $\mu_{01}=\left(M_{0} M_{1}\right) /\left(M_{0}+M_{1}\right)$ is the reduced mass. It is defined in terms of the renormalized masses $M_{0}$ and $M_{1}$ of the two particles. These masses can be extracted as usual from an exponential fit at large time distances of the two-point correlation functions $\left\langle\tilde{\phi}_{i}(t) \tilde{\phi}_{i}(0)\right\rangle \approx A_{1, i}\left(e^{-M_{i} t}+e^{-M_{i}(T-t)}\right)$ for $i=0,1$. To reduce the statistical error we average over all points with the same source sink separation.

\section{Numerical result}

\subsection{BH method}

We generate ensembles using the Metropolis-Hastings algorithm with bare masses $m_{0}=-4.925$ and $m_{1}=-4.85$, and for simplicity we choose $\lambda_{0}=\lambda_{1}=2 \mu=2.5$. The list of ensembles generated in this work with their corresponding measured values of the masses $M_{0}$ and $M_{1}$ are compiled in Table 1. In this model, as observed in previous investigations of the scalar theory [13], we do not see relevant effects of excited states in the two-point correlators, i.e., they are dominated by the ground state from the first time slice.

In the following we discuss three different strategies to extract the scattering length:

1. We attempt a direct fit of Eq. (3) the the data.

2. We include an overall constant in the fit to account for the $O\left(\left(t-t_{i}\right)^{0}\right)$ effect.

3. We make use of a shifted function at fixed $t_{i}$ and $t_{f}$, $\Delta_{t} C_{4}^{\mathrm{BH}}\left(t_{f}, t, t_{i}\right)=C_{4}^{\mathrm{BH}}\left(t_{f}, t+1, t_{i}\right)-C_{4}^{\mathrm{BH}}\left(t_{f}, t, t_{i}\right)$, which cancels the constant term. We then determine $a_{0}$ by fitting to

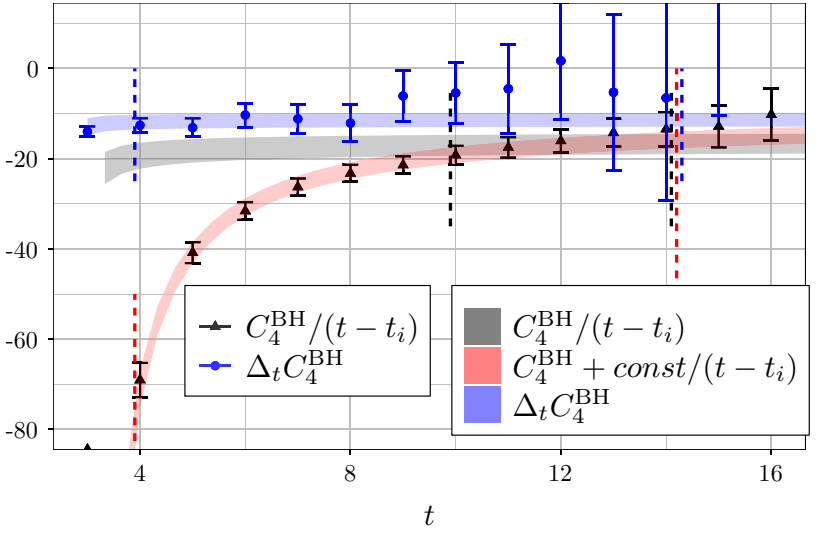

Fig. 1 Four-point function of Eq. (3) multiplied by $L^{3} / 2$, for $L=$ 22 and $T=96$ with $t_{i}=3$ and $t_{f}=16$ divided by $\left(t-t_{i}\right)$ black triangles. the dashed vertical lines represent the fit interval, the black band represent the result of the fit Eq. (3) and the red band is the same fit with an extra constant term. The blue circles and band represent the discrete derivative of the correlator Eq. (4) and the corresponding fit

$$
\begin{aligned}
& \Delta_{t} C_{4}^{\mathrm{BH}}\left(t_{f}, t, t_{i}\right) \approx \frac{2}{L^{3}}\left[\pi \frac{a_{0}}{\mu_{01}}-2 a_{0}^{2} \sqrt{\frac{2}{\mu_{01}}}\right. \\
& \left.\times\left(\sqrt{t+1-t_{i}}-\sqrt{t-t_{i}}\right)\right] .
\end{aligned}
$$

The three methods are compared in Fig. 1 for one of our ensembles. The black triangles represent the correlator of Eq. (3) divided by $\left(t-t_{i}\right)$ with $t_{i}=3$ and $t_{f}=16$. This representation is convenient, as it converges towards a constant when $\left(t-t_{i}\right) \rightarrow \infty$. From the monotonic increase of the data points, it is clear that the effect of the $\left(\left(t-t_{i}\right)^{0}\right)$ term in Eq. (3) is still sizeable even at large time separations. A fit in the time region $[10,14]$ — the black band-is reasonable $\left(\chi^{2} /\right.$ d.o.f $\left.\sim 0.7\right)$ but results in large uncertainties. The quality of the fit deteriorates very quickly if the fit range is extended: a fit in the time region $[6,14]$ yields a $\chi^{2} /$ dof $\sim 5$.

With the second strategy - the red band in Fig. 1-one is able to start fitting at significantly smaller $t$-values. The data are well described with a $\chi^{2} /$ dof $\sim 0.2$.

For the third approach, we study $\Delta_{t} C_{4}^{\mathrm{BH}}(t)$. This is shown in Fig. 1 as blue circles, and the blue band represents the best fit result with error. The main advantage of the last strategy is that it allows us to extract the physical information at smaller $t$ without introducing extra parameters in the fit. Indeed, the data looks almost constant over the complete $t$-range available. Only very close to $t_{i}$ the square root term might become visible.

For this third strategy, which looks most promising from a systematic point of view, we also investigate the dependence on the choice for $t_{i}$ and $t_{f}$. This is shown in Fig. 2 for the same ensemble as in Fig. 1. We do not observe any significant systematic effect stemming from excited state contributions 


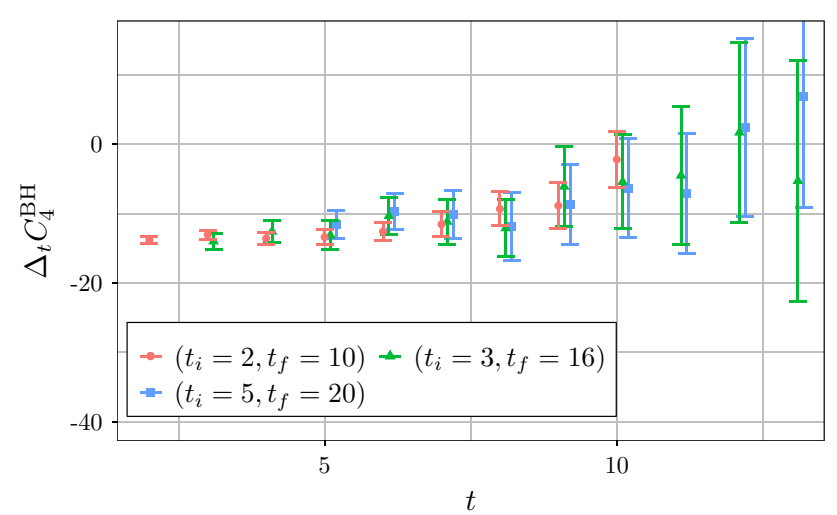

Fig. 2 Plot of the discrete derivative of the correlator Eq. (4) for different values of $t_{i}$ and $t_{f}$. We do not observe any systematic shift and all correlators are compatible. The points with smaller $t_{i}$ and $t_{f}$ tend to have smaller error

when changing $t_{i}$ or $t_{f}$. However, we clearly see significantly smaller statistical uncertainties with smaller $t_{i}$ and $t_{f}$ values.

\subsection{Comparison to the Lüscher method}

In this section we compare the $\mathrm{BH}$ method described above with the Lüscher threshold expansion [14,15]. The latter relates the two-particle energy shift, defined as $\Delta E_{2}=$ $E_{2}-M_{0}-M_{1}$, to the scattering length $a_{0}$ via

$\Delta E_{2}=-\frac{2 \pi a_{0}}{\mu_{01} L^{3}}\left[1+c_{1} \frac{a_{0}}{L}+c_{2}\left(\frac{a_{0}}{L}\right)^{2}\right]+O\left(L^{-6}\right)$,

with $c_{1}=-2.837297, c_{2}=6.375183$ and $E_{2}$ being the interacting two-particle energy. $E_{2}$ can be extracted from $C_{2}(t)=\left\langle\tilde{\phi}_{1}(t) \tilde{\phi}_{0}(t) \tilde{\phi}_{1}(0) \tilde{\phi}_{0}(0)\right\rangle$, whose large- $t$ behaviour is

$$
\begin{aligned}
& C_{2}(t) \underset{T-t \gg 0}{\stackrel{t \gg 0}{\longrightarrow}} A_{2} e^{-E_{2} \frac{T}{2}} \cosh \left(E_{2}\left(t-\frac{T}{2}\right)\right) \\
& \quad+B_{2} e^{-\left(M_{0}+M_{1}\right) \frac{T}{2}} \cosh \left(\left(M_{1}-M_{0}\right)\left(t-\frac{T}{2}\right)\right) .
\end{aligned}
$$

Note that the last term is a known thermal pollution due to finite $T$ in the presence of periodic boundary conditions. Using $M_{0}$ and $M_{1}$ as input determined from the corresponding two-point functions, the only additional parameter is $B_{2}$.

Alternatively, it is possible to eliminate the second term defining

$\tilde{C}_{2}(t)=C_{2}(t) / \cosh \left(\left(M_{1}-M_{0}\right)\left(t-\frac{T}{2}\right)\right)$,

and then taking the finite derivative

$$
\Delta_{t} \tilde{C}_{2}(t)=\tilde{C}_{2}(t+1)-\tilde{C}_{2}(t)
$$

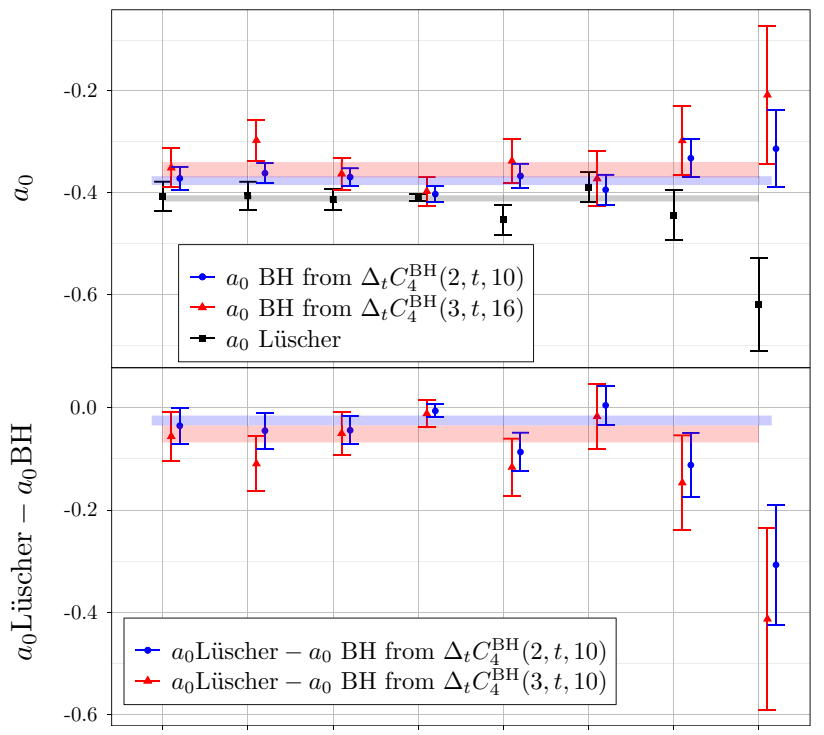

L20T48 L20T64 L20T96 L20T128 L22T96 L24T96 L26T96 L32T96

Fig. 3 Comparison of $a_{0}$ computed with BH method Eq. (4) with $t_{i}=$ 2 and $t_{f}=10$ (blue circles), with $t_{i}=3$ and $t_{f}=16$ (red triangles) and Lüscher method Eq. (5) (black squares) in the top panel. In the bottom panel we plot the correlated difference between Lüscher method and $\mathrm{BH}$ method. The horizontal bands correspond to the weighted average of each method

The two-particle energies obtained from Eq. (6) are compatible to those from Eq. (8). The results are reported in Table 1, along with the values for the scattering length $a_{0}$ computed from $E_{2}$ using Eq. (5). We have calculated the correlated difference between our two estimates of $a_{0}$ obtained within the Lüscher method, column 7 and 8 of Table 1 . We find that the difference is always compatible with zero within one sigma, with the exception of the ensembles L20T48 and L32T96 where it is within two sigma.

A comparison between the $\mathrm{BH}$ and the Lüscher method is depicted in Fig. 3 for all our ensembles. The values are compatible with each other, however the $\mathrm{BH}$ method gives systematically larger values for $a_{0}$. We do not observe a clear statistical correlation or anti correlation between the $\mathrm{BH}$ and the Lüscher method. We find that the correlation coefficient varies within the range $[-0.25,0.6]$ in all ensembles. We notice that exponentially suppressed finite-volume errors are in principle a source of systematic error. However, in Fig. 3, the determination of $a_{0}$ does not show any systematic effect varying $L$ from 20 to 32 .

\section{Conclusion}

In this letter, we have investigated the $\mathrm{BH}$ method, proposed in Ref. [1], using a scalar theory on the lattice. We have indeed verified that it is a viable method to obtain the scattering length, and that it produces results that are compatible with 
Table 1 Values of $a_{0}, M_{0}, M_{1}$ and $E_{2}$ measured. The column $\Delta_{t} C_{4}^{\mathrm{BH}}$ corresponds to the value of $a_{0}$ fitted with Eq. (4) fixing $t_{i}=3$ and $t_{f}=16$ or $t_{i}=2$ and $t_{f}=10$, the column $C_{\mathrm{BH}}+c$ is the result of the fit Eq. (3) adding a constant term. The two-particle energy $E_{2}$ is computed from $C_{2}$ with the fit of Eq. (6) and from $\Delta \tilde{C}_{2}$ with Eq. (8). The corresponding value of $a_{0}$ computed with the Lüscher method is reported in the corresponding columns. We used $2 \cdot 10^{7}$ configurations for each ensemble, generated from 200 replicas each of $10^{5}$ thermalized configurations, we bin the configurations in blocks of $10^{5}$ (the entirely replica) and we resample the resulting 200 configurations with jackknife. For the light mass $M_{0}$ we measured the integrated autocorrelation time $\tau_{\text {int }} \sim 1.5$, while $\tau_{\text {int }} \sim 0.5$ for $M_{1}$, we skip 1000 configurations in each replica for thermalization

\begin{tabular}{|c|c|c|c|c|c|c|c|c|c|c|}
\hline \multirow[t]{2}{*}{$\mathrm{T}$} & \multirow[t]{2}{*}{$\mathrm{L}$} & \multirow[t]{2}{*}{$M_{0}$} & \multirow[t]{2}{*}{$M_{1}$} & \multicolumn{2}{|l|}{$\underline{E_{2}}$} & \multicolumn{2}{|l|}{$a_{0}$ Lüscher } & \multicolumn{3}{|l|}{$a_{0} \mathrm{BH}$} \\
\hline & & & & $C_{2}$ & $\Delta_{t} \tilde{C}_{2}$ & $C_{2}$ & $\Delta_{t} \tilde{C}_{2}$ & $\Delta_{t} C_{4}^{\mathrm{BH}}(3, \mathrm{t}, 16)$ & $C_{4}^{\mathrm{BH}}+c$ & $\Delta_{t} C_{4}^{\mathrm{BH}}(2, \mathrm{t}, 10)$ \\
\hline 48 & 20 & $0.14675(5)$ & $0.27487(5)$ & $0.4252(3)$ & $0.4253(3)$ & $-0.41(3)$ & $-0.42(3)$ & $-0.35(4)$ & $-0.35(6)$ & $-0.37(2)$ \\
\hline 64 & 20 & $0.14659(5)$ & $0.27480(5)$ & $0.4249(3)$ & $0.4250(3)$ & $-0.41(3)$ & $-0.41(4)$ & $-0.30(4)$ & $-0.29(6)$ & $-0.38(2)$ \\
\hline 96 & 20 & $0.14662(4)$ & $0.27487(4)$ & $0.4251(2)$ & $0.4251(3)$ & $-0.41(2)$ & $-0.41(3)$ & $-0.36(3)$ & $-0.36(4)$ & $-0.38(1)$ \\
\hline 96 & 22 & $0.14604(3)$ & $0.27470(4)$ & $0.4237(2)$ & $0.4237(3)$ & $-0.45(3)$ & $-0.45(5)$ & $-0.34(4)$ & $-0.31(6)$ & $-0.37(2)$ \\
\hline 96 & 24 & $0.14574(4)$ & $0.27458(4)$ & $0.4223(2)$ & $0.4221(3)$ & $-0.39(3)$ & $-0.36(6)$ & $-0.36(5)$ & $-0.41(7)$ & $-0.39(2)$ \\
\hline 96 & 26 & $0.14547(4)$ & $0.27455(3)$ & $0.4218(2)$ & $0.4219(3)$ & $-0.44(5)$ & $-0.47(8)$ & $-0.30(7)$ & $-0.3(1)$ & $-0.36(3)$ \\
\hline 96 & 32 & $0.14521(4)$ & $0.27449(4)$ & $0.4210(2)$ & $0.4213(3)$ & $-0.62(9)$ & $-0.7(1)$ & $-0.2(1)$ & $-0.1(2)$ & $-0.35(5)$ \\
\hline 128 & 20 & $0.14668(3)$ & $0.27484(3)$ & $0.42509(7)$ & $0.4251(3)$ & $-0.409(7)$ & $-0.41(3)$ & $-0.40(3)$ & $-0.39(3)$ & $-0.40(1)$ \\
\hline
\end{tabular}

those of the Lüscher method [14]. The most reliable strategy to analyse the four-point function is found to be the use of finite differences in time to remove an overall constant term.

We observe a systematic difference between the Lüscher and $\mathrm{BH}$ method. Interestingly, for each ensemble separately both determinations appear compatible. The systematic trend becomes evident only after averaging over all runs, as shown in the bands of Fig. 3. This might be attributed to different lattice artefacts, since both methods represent different estimators for $a_{0}$. We are not able to check this hypothesis here, because we cannot take the continuum limit. However, the different systematics of the two methods offer in general a useful opportunity for cross checks.

The statistical error is similar in both approaches. Also the scaling in $L$ appears to be similar, with maybe a slight advantage for the $\mathrm{BH}$ method. However, any advantage of one method compared to another one will in general depend on the theory considered. We conclude that it seems promising to use the BH method in lattice QCD for instance for $\pi N$ scattering, where also the lattice spacing dependence could be investigated.

Acknowledgements We gratefully acknowledge helpful discussions with M. Bruno, M. T. Hansen and S. R. Sharpe. FRL acknowledges financial support from Generalitat Valenciana grants PROMETEO/2019/083 and CIDEGENT/2019/040, the EU project H2020MSCA-ITN-2019//860881-HIDDeN, and the Spanish project FPA201785985-P. This work is supported in part by the Deutsche Forschungsgemeinschaft (DFG, German Research Foundation) and the NSFC through the funds provided to the Sino-German Collaborative Research Center CRC 110 "Symmetries and the Emergence of Structure in QCD” (DFG Project-ID 196253076-TRR 110, NSFC Grant No. 12070131001). AR acknowledges support from Volkswagenstiftung (Grant No. 93562) and the Chinese Academy of Sciences (CAS) President's International Fellowship Initiative (PIFI) (Grant No. 2021VMB 0007). The $\mathrm{C}++$ Performance Portability Programming Model Kokkos
[16] and the open source software packages R [17] have been used. We thank B. Kostrzewa for useful discussions on Kokkos.

Data Availability Statement This manuscript has no associated data or the data will not be deposited. [Authors' comment: The data generated for this letter is available in form of correlation function upon request.]

Open Access This article is licensed under a Creative Commons Attribution 4.0 International License, which permits use, sharing, adaptation, distribution and reproduction in any medium or format, as long as you give appropriate credit to the original author(s) and the source, provide a link to the Creative Commons licence, and indicate if changes were made. The images or other third party material in this article are included in the article's Creative Commons licence, unless indicated otherwise in a credit line to the material. If material is not included in the article's Creative Commons licence and your intended use is not permitted by statutory regulation or exceeds the permitted use, you will need to obtain permission directly from the copyright holder. To view a copy of this licence, visit http://creativecomm ons.org/licenses/by/4.0/.

Funded by $\mathrm{SCOAP}^{3}$.

\section{References}

1. M. Bruno, M.T. Hansen, J. High Energy Phys. 2021(6) (2021). https://doi.org/10.1007/JHEP06(2021)043

2. M. Lüscher, U. Wolff, Nucl. Phys. B 339, 222 (1990)

3. R.A. Briceno, J.J. Dudek, R.D. Young, Rev. Mod. Phys. 90(2), 025001 (2018)

4. M.T. Hansen, S.R. Sharpe, Phys. Rev. D $90(11), 116003$ (2014)

5. M.T. Hansen, S.R. Sharpe, Phys. Rev. D 92(11), 114509 (2015)

6. H.W. Hammer, J.Y. Pang, A. Rusetsky, JHEP 09, 109 (2017)

7. H.W. Hammer, J.Y. Pang, A. Rusetsky, JHEP 10, 115 (2017)

8. M. Mai, M. Döring, Eur. Phys. J. A 53(12), 240 (2017)

9. M.T. Hansen, S.R. Sharpe, Annu. Rev. Nucl. Part. Sci. 69, 65 (2019)

10. M. Mai, M. Döring, A. Rusetsky, EPJ ST (2021)

11. S.R. Sharpe, Phys. Rev. D 96(5), 054515 (2017) [Erratum: Phys. Rev. D 98, 099901 (2018)]

12. F. Romero-López, A. Rusetsky, C. Urbach, Eur. Phys. J. C 78(10), 846 (2018) 
13. F. Romero-López, A. Rusetsky, N. Schlage, C. Urbach, JHEP 02, $060(2021)$

14. M. Lüscher, Commun. Math. Phys. 104, 177 (1986). http:// inspirehep.net/record/222569

15. S. Beane, P. Bedaque, A. Parreño, M. Savage, Nucl. Phys. A 747(1), 55-74 (2005). https://doi.org/10.1016/j.nuclphysa.2004.09.081
16. H.C. Edwards, C.R. Trott, D. Sunderland, J. Parallel Distrib. Comput. 74(12), 3202 (2014). http://www.sciencedirect.com/science/ article/pii/S0743731514001257

17. R Core Team, R: A Language and Environment for Statistical Computing. R Foundation for Statistical Computing, Vienna (2019). https://www.R-project.org/ 